\title{
A FRICTIONLESS CONTACT PROBLEM FOR VISCOELASTIC MATERIALS
}

\author{
MIKÄEL BARBOTEU, WEIMIN HAN, \\ AND MIRCEA SOFONEA
}

Received 12 March 2001 and in revised form 4 September 2001

We consider a mathematical model which describes the contact between a deformable body and an obstacle, the so-called foundation. The body is assumed to have a viscoelastic behavior that we model with the KelvinVoigt constitutive law. The contact is frictionless and is modeled with the well-known Signorini condition in a form with a zero gap function. We present two alternative yet equivalent weak formulations of the problem and establish existence and uniqueness results for both formulations. The proofs are based on a general result on evolution equations with maximal monotone operators. We then study a semi-discrete numerical scheme for the problem, in terms of displacements. The numerical scheme has a unique solution. We show the convergence of the scheme under the basic solution regularity. Under appropriate regularity assumptions on the solution, we also provide optimal order error estimates.

\section{Introduction}

Contact phenomena involving deformable bodies abound in industry and everyday life. The contact of the braking pads with the wheel, the tire with the road, and the piston with the skirt are just three simple examples. Despite the difficulties that the contact processes present because of the complicated surface phenomena involved, a considerable progress has been made in their modeling and analysis, and the literature in this field is extensive. For the sake of simplicity, we refer in the following only to results and references concerning frictionless contact problems. More details and bibliographical comments with regard to 
contact problems with or without friction can be found in the monograph [9], for instance.

The study of frictionless problems represents a first step in the study of more complicated contact problems, involving friction. The famous Signorini problem was formulated in [24] as a model of unilateral frictionless contact between an elastic body and a rigid foundation. Mathematical analysis of this problem was first provided in [6] and was subsequently published in full in [7]. In [18], numerical approximation of the problem is described in detail. An optimal order error estimate is derived for the linear element solution, under suitable solution regularity assumptions. Some solution algorithms for solving the finite-element system are introduced and discussed. Results concerning the frictionless Signorini contact problem between two elastic bodies have been obtained in [10,11, 12,13]. In these papers the authors consider two types of problems: (1) with a bounded zone of contact, when the zone of contact cannot enlarge during the deformation process; (2) with an increasing zone of contact, when the range of the contact zone may expand during the process. They present variational formulations of the problems in terms of displacement and stress, respectively, and provide existence and uniqueness results of the weak solutions; further, they consider a finite-element model for solving the contact problems, derive error estimates in the case of regular solutions, prove convergence results in the case of irregular solutions, and discuss some solution algorithms.

In all the references above, it was assumed that the deformable bodies were linearly elastic. However, a number of recent publications are dedicated to the modeling, analysis, and numerical approximation of contact problems involving viscoelastic and viscoplastic materials. For example, the variational analysis of the frictionless Signorini problem was provided in [25] in the case of rate-type viscoplastic materials and extended in [3] in the study of rate-type viscoplastic materials with internal state variables. The frictionless contact between two viscoplastic bodies was studied in [23] and the numerical analysis of this problem was performed in [8]. In all these papers, the processes were assumed to be quasistatic and the unique solvability of the corresponding contact problems has been obtained by using arguments on time-dependent elliptic variational inequalities and the Banach fixed-point theorem. A survey of these results, including numerical experiments for test problems in one, two, and three dimensions, may be found in $[5,9]$. Existence results in the study of the dynamic Signorini frictionless contact problem for viscoelastic materials with singular memory have been obtained in [16, 17]. 
The aim of this paper is to present new results in the study of the frictionless Signorini problem. We consider here quasistatic processes for Kelvin-Voigt viscoelastic materials in which the elasticity operator may be nonlinear. We derive two alternative yet equivalent weak formulations of the problem, which lead to evolutionary systems for the displacement and stress field. Then, we prove the unique solvability of the systems and therefore we deduce the existence of the unique weak solution to the frictionless contact problem. We also discuss the numerical treatment of the problem, based on a spatially semi-discrete scheme for the displacement field, and derive error estimates and convergence results.

The paper is organized as follows. In Section 2, we state the mechanical problem and present the notation and preliminary material. In Section 3, we list the assumptions imposed on the problem data and derive two variational formulations to the model. We show the unique solvability and the equivalence of the variational formulations in Section 4. The proofs are based on an abstract result on evolution equations with maximal monotone operators and arguments on convex analysis. In Section 5, we analyze a semi-discrete scheme, employing the finiteelement method to discretize the spatial domain. We show the existence of a unique numerical solution, prove convergence of the numerical solution, and derive error estimates under additional solution regularity.

\section{Problem statement and preliminaries}

We consider a viscoelastic body which occupies a domain $\Omega \subset \mathbb{R}^{d}(d \leq 3$ in applications) with outer Lipschitz surface $\Gamma$ that is divided into three disjoint measurable parts $\Gamma_{i}, i=1,2,3$, such that meas $\left(\Gamma_{1}\right)>0$. Let $[0, T]$ be the time interval of interest, where $T>0$, and let $\boldsymbol{v}$ denote the unit outer normal on $\Gamma$. The body is clamped on $\Gamma_{1} \times(0, T)$ and therefore the displacement field vanishes there. A volume force of density $\mathbf{f}_{0}$ acts in $\Omega \times(0, T)$ and surface tractions of density $\mathbf{f}_{2}$ act on $\Gamma_{2} \times(0, T)$. We assume that the body forces and tractions vary slowly with time, so the inertial terms may be neglected in the equation of motion, leading to a quasistatic problem. The body is in contact on $\Gamma_{3} \times(0, T)$ with a rigid obstacle, the so-called foundation. The contact is frictionless and it is modeled with the Signorini contact conditions, in the form with a zero gap function.

With these assumptions, denoting by $\mathbb{S}^{d}$ the space of second-order symmetric tensors on $\mathbb{R}^{d}$, the classical formulation of the frictionless contact problem of the viscoelastic body is the following. 
4 A frictionless contact problem for viscoelastic materials

Problem 2.1. Find a displacement field $\mathbf{u}: \Omega \times[0, T] \rightarrow \mathbb{R}^{d}$ and a stress field $\sigma: \Omega \times[0, T] \rightarrow \mathbb{S}^{d}$ such that

$$
\begin{aligned}
\boldsymbol{\sigma} & =\mathcal{A} \boldsymbol{\varepsilon}(\dot{\mathbf{u}})+\mathcal{G} \varepsilon(\mathbf{u}) & & \text { in } \Omega \times(0, T), \\
\operatorname{Div} \boldsymbol{\sigma}+\mathbf{f}_{0} & =\mathbf{0} & & \text { in } \Omega \times(0, T), \\
\mathbf{u} & =\mathbf{0} & & \text { on } \Gamma_{1} \times(0, T), \\
\boldsymbol{\sigma} \boldsymbol{v} & =\mathbf{f}_{2} & & \text { on } \Gamma_{2} \times(0, T), \\
u_{v} \leq 0, \quad \sigma_{v} & \leq 0, \quad \sigma_{v} u_{v}=0, \quad \boldsymbol{\sigma}_{\tau}=\mathbf{0} & & \text { on } \Gamma_{3} \times(0, T), \\
\mathbf{u}(0) & =\mathbf{u}_{0} & & \text { in } \Omega .
\end{aligned}
$$

In (2.1), (2.2), (2.3), (2.4), (2.5), and (2.6) and below, in order to simplify the notation, we do not indicate explicitly the dependence of various functions on the variables $\mathbf{x} \in \Omega \cup \Gamma$ and $t \in[0, T]$. Equation (2.1) represents the viscoelastic constitutive law in which $\mathcal{A}$ is a fourth-order tensor, $\mathcal{G}$ is a nonlinear constitutive function, and $\boldsymbol{\varepsilon}(\mathbf{u})$ denotes the small strain tensor. Here and everywhere in this paper, the dot represents the derivative with respect to the time variable. Equation (2.2) is the equilibrium equation, while conditions (2.3) and (2.4) are the displacement and traction boundary conditions, respectively. Conditions (2.5) represent the frictionless Signorini contact conditions in which $u_{v}$ denotes the normal displacement, $\sigma_{v}$ represents the normal stress, and $\boldsymbol{\sigma}_{\tau}$ is the tangential stress on the potential contact surface. Finally, (2.6) represents the initial condition in which $\mathbf{u}_{0}$ is the initial displacement field.

Kelvin-Voigt viscoelastic materials of the form (2.1) involving nonlinear constitutive functions have been considered recently in [21, 22]. We recall that in linear viscoelasticity, the stress tensor $\sigma=\left(\sigma_{i j}\right)$ is given by

$$
\sigma_{i j}=a_{i j k h} \varepsilon_{k h}(\dot{\mathbf{u}})+g_{i j k h} \varepsilon_{k h}(\mathbf{u}),
$$

where $\mathcal{A}=\left(a_{i j k h}\right)$ is the viscosity tensor and $\mathcal{G}=\left(g_{i j k h}\right)$ is the elasticity tensor. Here and below the indices $i, j, k, h$ run between 1 and $d$ and the summation convention over repeated indices is adopted.

We now make some comments on the Signorini contact conditions (2.5) in which our interest is. When equality $u_{v}=0$ holds, there is a contact between the body and the foundation and when inequality $u_{v}<0$ holds, there is no contact. Therefore, at each time instant, the surface $\Gamma_{3}$ is divided into two zones: the zone of contact and the zone of separation. The boundary of these zones is the free boundary, since they are unknown a priori and are part of the problem. However, a key limitation of problems (2.1), (2.2), (2.3), (2.4), (2.5), and (2.6) is that the potential contact surface $\Gamma_{3}$ is assumed to be known a priori. Considering the case when the potential contact surface is not known and may enlarge during 
the deformation process (cf. $[10,13]$ ) leads to substantial mathematical difficulties and it is left open.

To study the mechanical problems (2.1), (2.2), (2.3), (2.4), (2.5), and (2.6) we introduce the notation we will use and some preliminary material. For further details, we refer the reader to $[4,15,20]$. We denote by "." and $|\cdot|$ the inner product and the Euclidean norm on $\mathbb{S}^{d}$ and $\mathbb{R}^{d}$, respectively, that is,

$$
\begin{aligned}
& \mathbf{u} \cdot \mathbf{v}=u_{i} v_{i}, \quad|\mathbf{v}|=(\mathbf{v} \cdot \mathbf{v})^{1 / 2} \quad \forall \mathbf{u}, \mathbf{v} \in \mathbb{R}^{d}, \\
& \boldsymbol{\sigma} \cdot \boldsymbol{\tau}=\sigma_{i j} \tau_{i j}, \quad|\boldsymbol{\tau}|=(\boldsymbol{\tau} \cdot \boldsymbol{\tau})^{1 / 2} \quad \forall \boldsymbol{\sigma}, \boldsymbol{\tau} \in \mathbb{S}^{d} .
\end{aligned}
$$

We will use the spaces

$$
\begin{aligned}
H & =L^{2}(\Omega)^{d}=\left\{\mathbf{u}=\left(u_{i}\right) \mid u_{i} \in L^{2}(\Omega)\right\}, \\
Q & =\left\{\boldsymbol{\sigma}=\left(\sigma_{i j}\right) \mid \sigma_{i j}=\sigma_{j i} \in L^{2}(\Omega)\right\}, \\
H_{1} & =\left\{\mathbf{u}=\left(u_{i}\right) \in H \mid \boldsymbol{\varepsilon}(\mathbf{u}) \in Q\right\}, \\
Q_{1} & =\{\boldsymbol{\sigma} \in Q \mid \operatorname{Div} \boldsymbol{\sigma} \in H\} .
\end{aligned}
$$

Here $\varepsilon: H_{1} \rightarrow Q$ and Div : $Q_{1} \rightarrow H$ are the deformation and divergence operators, respectively, defined by

$$
\boldsymbol{\varepsilon}(\mathbf{u})=\left(\varepsilon_{i j}(\mathbf{u})\right), \quad \varepsilon_{i j}(\mathbf{u})=\frac{1}{2}\left(u_{i, j}+u_{j, i}\right), \quad \operatorname{Div} \boldsymbol{\sigma}=\left(\sigma_{i j, j}\right),
$$

where the index that follows a comma indicates a partial derivative with respect to the corresponding component of the independent variable. The spaces $H, Q, H_{1}$, and $Q_{1}$ are real Hilbert spaces endowed with the canonical inner products given by

$$
\begin{aligned}
(\mathbf{u}, \mathbf{v})_{H} & =\int_{\Omega} u_{i} v_{i} d x \\
(\boldsymbol{\sigma}, \boldsymbol{\tau})_{Q} & =\int_{\Omega} \sigma_{i j} \tau_{i j} d x \\
(\mathbf{u}, \mathbf{v})_{H_{1}} & =(\mathbf{u}, \mathbf{v})_{H}+(\boldsymbol{\varepsilon}(\mathbf{u}), \boldsymbol{\varepsilon}(\mathbf{v}))_{Q^{\prime}} \\
(\boldsymbol{\sigma}, \boldsymbol{\tau})_{Q_{1}} & =(\boldsymbol{\sigma}, \boldsymbol{\tau})_{Q}+(\operatorname{Div} \boldsymbol{\sigma}, \operatorname{Div} \boldsymbol{\tau})_{H}
\end{aligned}
$$

The associated norms on these spaces are denoted by $\|\cdot\|_{H},\|\cdot\|_{Q},\|\cdot\|_{H_{1}}$, and $\|\cdot\|_{Q_{1}}$, respectively.

For every element $\mathbf{v} \in H_{1}$, we still write $\mathbf{v}$ for the trace $\gamma \mathbf{v}$ of $\mathbf{v}$ on $\Gamma$ and we denote by $v_{v}$ and $\mathbf{v}_{\tau}$ the normal and tangential components of $\mathbf{v}$ on the boundary $\Gamma$ given by

$$
v_{v}=\mathbf{v} \cdot \boldsymbol{v}, \quad \mathbf{v}_{\tau}=\mathbf{v}-v_{v} \boldsymbol{v}
$$


6 A frictionless contact problem for viscoelastic materials

For a regular (say $C^{1}$ ) stress field $\sigma$, the application of its trace on the boundary to $\boldsymbol{v}$ is the Cauchy stress vector $\boldsymbol{\sigma} \boldsymbol{v}$. We define, similarly, the normal and tangential components of the stress on the boundary by the formulas

$$
\sigma_{v}=(\boldsymbol{\sigma} \boldsymbol{v}) \cdot \boldsymbol{v}, \quad \sigma_{\tau}=\boldsymbol{\sigma} \boldsymbol{v}-\sigma_{v} \boldsymbol{v},
$$

and we recall that the following Green's formula holds:

$$
(\boldsymbol{\sigma}, \boldsymbol{\varepsilon}(\mathbf{v}))_{Q}+(\operatorname{Div} \boldsymbol{\sigma}, \mathbf{v})_{H}=\int_{\Gamma} \boldsymbol{\sigma} \boldsymbol{v} \cdot \mathbf{v} d a \quad \forall \mathbf{v} \in H_{1} .
$$

Keeping in mind the boundary conditions (2.3) and (2.5), we introduce the closed subspace of $H_{1}$ defined by

$$
V=\left\{\mathbf{v} \in H_{1} \mid \mathbf{v}=\mathbf{0} \text { on } \Gamma_{1}\right\}
$$

and the set of admissible displacement fields given by

$$
K=\left\{\mathbf{v} \in V \mid v_{v} \leq 0 \text { on } \Gamma_{3}\right\} .
$$

Since meas $\left(\Gamma_{1}\right)>0$, Korn's inequality holds: there exists $C_{K}>0$ which depends only on $\Omega$ and $\Gamma_{1}$ such that

$$
\|\varepsilon(\mathbf{v})\|_{Q} \geq C_{K}\|\mathbf{v}\|_{H_{1}} \quad \forall \mathbf{v} \in V .
$$

A proof of Korn's inequality (2.17) may be found in [19, page 79].

Finally, for every real Hilbert space $X$, we use the classical notation for the spaces $L^{p}(0, T, X)$ and $W^{k, p}(0, T, X), 1 \leq p \leq+\infty, k=1,2, \ldots$.

We will need the following result for existence proofs.

Theorem 2.2. Let $X$ be a real Hilbert space and let $A: D(A) \subset X \rightarrow 2^{X}$ be a multivalued operator such that the operator $A+\omega I$ is maximal monotone for some real $\omega$. Then, for every $f \in W^{1,1}(0, T ; X)$ and $u_{0} \in D(A)$, there exists a unique function $u \in W^{1, \infty}(0, T ; X)$ which satisfies

$$
\dot{u}(t)+A u(t) \ni f(t) \quad \text { a.e. } t \in(0, T), \quad u(0)=u_{0} .
$$

A proof of Theorem 2.2 may be found in [1, page 32]. Here and below $D(A)$ denotes the domain of the multivalued operator $A, 2^{X}$ represents the set of the subsets of $X$, and $I$ is the identity map on $X$.

\section{Variational formulations}

In this section, we list the assumptions imposed on the data, derive variational formulations of the mechanical problem, and state well-posedness results. 
We assume that the viscosity tensor $\mathcal{A}=\left(a_{i j k h}\right): \Omega \times \mathbb{S}^{d} \rightarrow \mathbb{S}^{d}$ satisfies the usual properties of symmetry and ellipticity

$$
\begin{aligned}
& a_{i j k h} \in L^{\infty}(\Omega) ; \\
& \mathcal{A} \boldsymbol{\sigma} \cdot \boldsymbol{\tau}=\boldsymbol{\sigma} \cdot \mathscr{A} \boldsymbol{\tau} \quad \forall \boldsymbol{\sigma}, \boldsymbol{\tau} \in \mathbb{S}^{d}, \text { a.e. in } \Omega ; \\
& \exists m_{\mathcal{A}}>0: \mathcal{A} \boldsymbol{\tau} \cdot \boldsymbol{\tau} \geq m_{\mathscr{A}}|\boldsymbol{\tau}|^{2} \quad \forall \boldsymbol{\tau} \in \mathbb{S}^{d}, \text { a.e. in } \Omega .
\end{aligned}
$$

The elasticity operator $\mathcal{G}: \Omega \times \mathbb{S}^{d} \rightarrow \mathbb{S}^{d}$ satisfies

$$
\begin{aligned}
& \exists \mathcal{L}_{\mathcal{G}}>0 \text { such that }\left|\mathcal{G}\left(\mathbf{x}, \varepsilon_{1}\right)-\mathcal{G}\left(\mathbf{x}, \varepsilon_{2}\right)\right| \leq \mathcal{L}_{\mathcal{G}}\left|\varepsilon_{1}-\varepsilon_{2}\right| \\
& \qquad \forall \varepsilon_{1}, \varepsilon_{2} \in \mathbb{S}^{d} \text {, a.e. } \mathbf{x} \in \Omega ; \\
& \mathbf{x} \longmapsto \mathcal{G}(\mathbf{x}, \boldsymbol{\varepsilon}) \text { is Lebesgue measurable on } \Omega \quad \forall \varepsilon \in \mathbb{S}^{d} ; \\
& \mathbf{x} \longmapsto \mathcal{G}(\mathbf{x}, 0) \in Q .
\end{aligned}
$$

Clearly, assumptions (3.1) and (3.2) are satisfied for the linear viscoElastic model (2.7) if the components $a_{i j k l}$ and $g_{i j k l}$ belong to $L^{\infty}(\Omega)$ and satisfy the usual properties of symmetry and ellipticity. A second example is provided by the nonlinear viscoelastic constitutive law

$$
\boldsymbol{\sigma}=\mathcal{A} \dot{\varepsilon}+\beta\left(\varepsilon-p_{K} \varepsilon\right) .
$$

Here $\mathcal{A}$ is a fourth-order tensor which satisfies (3.1), $\beta>0, K$ is a closedconvex subset of $\mathbb{S}^{d}$ such that $0 \in K$ and $p_{K}: \mathbb{S}^{d} \rightarrow K$ denotes the projection map. Using the nonexpansivity of the projection, we see that the elasticity operator $\mathcal{G}(\mathbf{x}, \varepsilon)=\beta\left(\varepsilon-D_{K} \varepsilon\right)$ satisfies condition (3.2). We conclude that the results below are valid for Kelvin-Voigt viscoelastic materials of the form (2.7) and (3.3), under the above assumptions.

We suppose that the body forces and surface tractions have the regularity

$$
\mathbf{f}_{0} \in W^{1,1}(0, T ; H), \quad \mathbf{f}_{2} \in W^{1,1}\left(0, T ; L^{2}\left(\Gamma_{2}\right)^{d}\right),
$$

and, finally, the initial displacement satisfies

$$
\mathbf{u}_{0} \in K
$$

For $\mathbf{u}, \mathbf{v} \in V$ let

$$
(\mathbf{u}, \mathbf{v})_{V}=(\mathcal{A} \varepsilon(\mathbf{u}), \varepsilon(\mathbf{v}))_{Q^{\prime}} \quad\|\mathbf{u}\|_{V}=(\mathbf{u}, \mathbf{u})_{V}^{1 / 2}
$$

Using (3.1) and (2.17) we obtain that $(\cdot, \cdot)_{V}$ is an inner product on $V$ and $\|\cdot\|_{V}$ and $\|\cdot\|_{H_{1}}$ are equivalent norms on $V$. Therefore, $\left(V,\|\cdot\|_{V}\right)$ is a real Hilbert space. 
8 A frictionless contact problem for viscoelastic materials

Next, we denote by $\mathbf{f}(t)$ the element of $V$ given by

$$
(\mathbf{f}(t), \mathbf{v})_{V}=\left(\mathbf{f}_{0}(t), \mathbf{v}\right)_{H}+\left(\mathbf{f}_{2}(t), \mathbf{v}\right)_{L^{2}\left(\Gamma_{2}\right)^{d}} \quad \forall \mathbf{v} \in V, t \in[0, T],
$$

and we note that conditions (3.4) imply

$$
\mathbf{f} \in W^{1,1}(0, T ; V) .
$$

Finally, for a.e. $t \in[0, T]$, we denote the set of admissible stress fields given by

$$
\Sigma(t)=\left\{\boldsymbol{\tau} \in Q \mid(\boldsymbol{\tau}, \boldsymbol{\varepsilon}(\mathbf{v}))_{Q} \geq(\mathbf{f}(t), \mathbf{v})_{V} \forall \mathbf{v} \in K\right\} .
$$

Using (2.12), (2.13), and (2.14), it is straightforward to show that if $\mathbf{u}$ and $\sigma$ are two regular functions satisfying (2.2), (2.3), (2.4), and (2.5), then $\mathbf{u}(t) \in V, \boldsymbol{\sigma}(t) \in Q_{1}$, and

$$
\mathbf{u}(t) \in K, \quad(\boldsymbol{\sigma}(t), \boldsymbol{\varepsilon}(\mathbf{v})-\boldsymbol{\varepsilon}(\mathbf{u}(t)))_{Q} \geq(\mathbf{f}(t), \mathbf{v}-\mathbf{u}(t))_{V} \quad \forall \mathbf{v} \in K, t \in[0, T] .
$$

Taking now $\mathbf{v}=2 \mathbf{u}(t)$ and $\mathbf{v}=\mathbf{0}$ in (3.10) we find

$$
\boldsymbol{\sigma}(t) \in \Sigma(t), \quad(\boldsymbol{\tau}-\boldsymbol{\sigma}(t), \boldsymbol{\varepsilon}(\mathbf{u}(t)))_{Q} \geq 0 \quad \forall \boldsymbol{\tau} \in \Sigma(t), t \in[0, T] .
$$

Inequalities (3.10), (3.11), combined with (2.1), (2.6), lead us to consider the following two variational problems.

Problem 3.1. Find a displacement field $\mathbf{u}:[0, T] \rightarrow V$ and a stress field $\sigma:[0, T] \rightarrow Q_{1}$ such that

$$
\begin{aligned}
\boldsymbol{\sigma}(t) & =\mathcal{A} \boldsymbol{\varepsilon}(\dot{\mathbf{u}}(t))+\mathcal{G} \varepsilon(\mathbf{u}(t)) \quad \text { a.e. } t \in(0, T), \\
\mathbf{u}(t) & \in K, \quad(\boldsymbol{\sigma}(t), \varepsilon(\mathbf{v})-\boldsymbol{\varepsilon}(\mathbf{u}(t)))_{Q} \geq(\mathbf{f}(t), \mathbf{v}-\mathbf{u}(t))_{V} \\
& \forall \mathbf{v} \in K, \text { a.e. } t \in(0, T), \\
\mathbf{u}(0) & =\mathbf{u}_{0} .
\end{aligned}
$$

Problem 3.2. Find a displacement field $\mathbf{u}:[0, T] \rightarrow V$ and stress field $\boldsymbol{\sigma}:$ $[0, T] \rightarrow Q_{1}$ which satisfy (3.12), (3.14), and

$$
\boldsymbol{\sigma}(t) \in \Sigma(t), \quad(\boldsymbol{\tau}-\boldsymbol{\sigma}(t), \varepsilon(\mathbf{u}(t)))_{Q} \geq 0 \quad \forall \boldsymbol{\tau} \in \Sigma(t) \text {, a.e. } t \in(0, T) .
$$

We remark that Problems 3.1 and 3.2 are formally equivalent to the mechanical problems (2.1), (2.2), (2.3), (2.4), (2.5), and (2.6). Indeed, if $\{\mathbf{u}, \boldsymbol{\sigma}\}$ represents a regular solution of the variational problem 3.1 or 3.2 , using the arguments of [4], it follows that $\{\mathbf{u}, \boldsymbol{\sigma}\}$ satisfies (2.1), (2.2), (2.3), (2.4), (2.5), and (2.6). For this reason, we may consider Problems 3.1 and 3.2 as variational formulations of the mechanical problems (2.1), (2.2), (2.3), (2.4), (2.5), and (2.6). 


\section{Existence and uniqueness results}

The main results of this section concern the unique solvability and the equivalence of the variational problems 3.1 and 3.2. We have the following results.

Theorem 4.1. Assume (3.1), (3.2), (3.4), and (3.5). Then there exists a unique solution $\{\mathbf{u}, \boldsymbol{\sigma}\}$ to Problem 3.1. Moreover, the solution satisfies

$$
\mathbf{u} \in W^{1, \infty}(0, T ; V), \quad \boldsymbol{\sigma} \in L^{\infty}\left(0, T ; Q_{1}\right) .
$$

Theorem 4.2. Assume (3.1), (3.2), (3.4), and (3.5) and let $\{\mathbf{u}, \boldsymbol{\sigma}\}$ be a couple of functions which satisfies (4.1). Then $\{\mathbf{u}, \boldsymbol{\sigma}\}$ is a solution of the variational Problem 3.1 if and only if $\{\mathbf{u}, \boldsymbol{\sigma}\}$ is a solution of the variational Problem 3.2.

Theorem 4.3. Assume (3.1), (3.2), (3.4), and (3.5). Then there exists a unique solution $\{\mathbf{u}, \boldsymbol{\sigma}\}$ to Problem 3.2. Moreover, the solution satisfies (4.1).

Theorems 4.1 and 4.3 state the unique solvability of Problems 3.1 and 3.2 , respectively, while Theorem 4.2 expresses the equivalence of these variational problems. From these theorems we conclude that the mechanical problem (2.1), (2.2), (2.3), (2.4), (2.5), and (2.6) has a unique weak solution which solves both Problems 3.1 and 3.2. Moreover, since Theorem 4.3 is a consequence of Theorems 4.1 and 4.2, we only need to provide the proofs of Theorems 4.1 and 4.2 .

We start with the proof of Theorem 4.1. We will apply Theorem 2.2.

Proof of Theorem 4.1. By the Riesz representation theorem we can define an operator $B: V \rightarrow V$ by

$$
(B \mathbf{u}, \mathbf{v})_{V}=(\mathcal{G} \varepsilon(\mathbf{u}), \varepsilon(\mathbf{v}))_{Q} \quad \forall \mathbf{u}, \mathbf{v} \in V .
$$

It follows from (3.2), (3.1), and (3.6) that

$$
\left\|B \mathbf{u}_{1}-B \mathbf{u}_{2}\right\|_{V} \leq \frac{\mathcal{L}_{\mathcal{G}}}{m_{\AA}}\left\|\mathbf{u}_{1}-\mathbf{u}_{2}\right\|_{V} \quad \forall \mathbf{u}_{1}, \mathbf{u}_{2} \in V,
$$

that is, $B$ is a Lipschitz continuous operator. Moreover, the operator

$$
B+\frac{\mathcal{L}_{\mathcal{G}}}{m_{A}} I: V \longrightarrow V
$$

is a monotone Lipschitz continuous operator on $V$. Let $\psi_{K}: V \rightarrow(-\infty$, $+\infty$ ] denote the indicator function of the set $K$ and let $\partial \psi_{K}$ be the subdifferential of $\psi_{K}$. Since $K$ is a nonempty, convex, closed part of $V$, it follows that $\partial \psi_{K}$ is a maximal monotone operator on $V$ and $D\left(\partial \psi_{K}\right)=K$. 
10 A frictionless contact problem for viscoelastic materials

Moreover, the sum

$$
\partial \psi_{K}+B+\frac{\mathcal{L}_{\mathcal{G}}}{m_{A}} I: K \subset V \longrightarrow 2^{V}
$$

is a maximal monotone operator. Thus, conditions (3.5) and (3.8) allow us to apply Theorem 2.2 with $X=V, A=\partial \psi_{K}+B: D(A)=K \subset V \rightarrow$ $2^{V}$, and $\omega=\mathcal{L}_{\mathcal{G}} / m_{\AA}$. We deduce that there exists a unique element $\mathbf{u} \in$ $W^{1, \infty}(0, T ; V)$ such that

$$
\begin{aligned}
\dot{\mathbf{u}}(t)+\partial \psi_{K}(\mathbf{u}(t))+B \mathbf{u}(t) & \ni \mathbf{f}(t) \quad \text { a.e. } t \in(0, T), \\
\mathbf{u}(0) & =\mathbf{u}_{0} .
\end{aligned}
$$

Since for any elements $\mathbf{u}, \mathbf{g} \in V$, the following equivalence holds:

$$
\mathbf{g} \in \partial \psi_{K}(\mathbf{u}) \Longleftrightarrow \mathbf{u} \in K, \quad(\mathbf{g}, \mathbf{v}-\mathbf{u})_{V} \leq 0 \quad \forall \mathbf{v} \in K,
$$

the differential inclusion (4.6) is equivalent to the following variational inequality:

$$
\begin{gathered}
\mathbf{u}(t) \in K, \quad(\dot{\mathbf{u}}(t), \mathbf{v}-\mathbf{u}(t))_{V}+(B \mathbf{u}(t), \mathbf{v}-\mathbf{u}(t))_{V} \geq(\mathbf{f}(t), \mathbf{v}-\mathbf{u}(t))_{V} \\
\forall \mathbf{v} \in K, \text { a.e. } t \in(0, T) .
\end{gathered}
$$

It follows now from (4.9), (4.2), and (3.6) that $\mathbf{u}$ satisfies the inequality

$$
\begin{aligned}
& \mathbf{u}(t) \in K, \quad(\mathcal{A} \varepsilon(\dot{\mathbf{u}}(t)),\varepsilon(\mathbf{v})-\varepsilon(\mathbf{u}(t)))_{Q}+(\mathcal{G} \varepsilon(\mathbf{u}(t)), \varepsilon(\mathbf{v})-\varepsilon(\mathbf{u}(t)))_{Q} \\
& \geq(\mathbf{f}(t), \mathbf{v}-\mathbf{u}(t))_{V} \quad \forall \mathbf{v} \in K, \text { a.e. } t \in(0, T) .
\end{aligned}
$$

Let $\sigma$ denote the function defined by (3.12). It follows from (4.10) and (4.7) that $\{\mathbf{u}, \boldsymbol{\sigma}\}$ is a solution of Problem 3.1. Moreover, since $\mathbf{u} \in$ $W^{1, \infty}(0, T ; V)$, from (3.12), (3.1), and (3.2) we obtain $\sigma \in L^{\infty}(0, T ; Q)$. Taking $\mathbf{v}=\mathbf{u}(t) \pm \boldsymbol{\varphi}$ in (3.13) where $\boldsymbol{\varphi} \in \boldsymbol{\Phi}(\Omega)^{d}$ and using (3.7), we find

$$
\operatorname{Div} \boldsymbol{\sigma}(t)+\mathbf{f}_{0}(t)=\mathbf{0} \quad \text { a.e. } t \in(0, T) .
$$

Keeping in mind (3.4), we obtain $\operatorname{Div} \sigma \in L^{\infty}(0, T ; H)$. Therefore, we deduce that $\sigma \in L^{\infty}\left(0, T ; Q_{1}\right)$ which concludes the existence part in Theorem 4.1 .

The uniqueness part results from the uniqueness of the element $\mathbf{u} \in$ $W^{1, \infty}(0, T ; V)$ which satisfies $(4.6),(4.7)$, guaranteed by Theorem 2.2.

Under the assumption

$$
\boldsymbol{\sigma} \boldsymbol{v} \in L^{\infty}\left(0, T ; L^{2}(\Gamma)^{d}\right)
$$


by a standard procedure (cf. [18]) we have

$$
\boldsymbol{\sigma} \boldsymbol{v}=\mathbf{f}_{2} \quad \text { a.e. on } \Gamma_{2} \times(0, T), \quad \boldsymbol{\sigma}_{\tau}=\mathbf{0} \quad \text { a.e. on } \Gamma_{3} \times(0, T) .
$$

These relations will be needed in error estimation of numerical solutions.

Proof of Theorem 4.2. Let $\{\mathbf{u}, \boldsymbol{\sigma}\}$ be a couple of functions which satisfies (4.1). We need to prove the equivalence of the inequalities (3.13) and (3.15). All the equalities, inequalities, and inclusions below involving the argument $t$ are understood to be valid for almost any $t \in(0, T)$.

Suppose that $\{\mathbf{u}, \boldsymbol{\sigma}\}$ satisfies (3.13). Choosing $\mathbf{v}=2 \mathbf{u}(t)$ and $\mathbf{v}=\mathbf{0}$ in (3.13) we find

$$
(\boldsymbol{\sigma}(t), \varepsilon(\mathbf{u}(t)))_{Q}=(\mathbf{f}(t), \mathbf{u})_{V} .
$$

Using now (3.13) and (4.14), we deduce that

$$
(\boldsymbol{\sigma}(t), \boldsymbol{\varepsilon}(\mathbf{v}))_{Q} \geq(\mathbf{f}(t), \mathbf{v})_{V} \quad \forall \mathbf{v} \in K,
$$

that is, $\sigma(t) \in \Sigma(t)$. The inequality in (3.15) follows now from (3.9) and (4.14), which concludes the first part of the proof.

Conversely, suppose that $\{\mathbf{u}, \boldsymbol{\sigma}\}$ satisfies (3.15). We will first prove that $\mathbf{u}(t) \in K$. Indeed, suppose that $\mathbf{u}(t) \notin K$ and denote by $P(\mathbf{u}(t))$ the projection of $\mathbf{u}(t)$ on the closed convex subset $K \subset V$. We have

$$
\begin{aligned}
& (P \mathbf{u}(t)-\mathbf{u}(t), \mathbf{v})_{V} \\
& \quad \geq(P \mathbf{u}(t)-\mathbf{u}(t), P \mathbf{u}(t))_{V}>(P \mathbf{u}(t)-\mathbf{u}(t), \mathbf{u}(t))_{V} \quad \forall \mathbf{v} \in K .
\end{aligned}
$$

From these inequalities we obtain that there exists $\alpha \in \mathbb{R}$ such that

$$
(P \mathbf{u}(t)-\mathbf{u}(t), \mathbf{v})_{V}>\alpha>(P \mathbf{u}(t)-\mathbf{u}(t), \mathbf{u}(t))_{V} \quad \forall \mathbf{v} \in K .
$$

Let $\tilde{\boldsymbol{\tau}}(t) \in Q$ be the element

$$
\tilde{\boldsymbol{\tau}}(t)=\mathcal{A} \boldsymbol{\varepsilon}(P \mathbf{u}(t)-\mathbf{u}(t)) .
$$

Using (3.6), (4.17), and (4.18) we deduce that

$$
(\tilde{\boldsymbol{\tau}}(t), \varepsilon(\mathbf{v}))_{Q}>\alpha>(\tilde{\boldsymbol{\tau}}(t), \varepsilon(\mathbf{u}(t)))_{Q} \quad \forall \mathbf{v} \in K
$$

and, taking $\mathbf{v}=\mathbf{0}$ in (4.19), we obtain

$$
\alpha<0 .
$$

Now suppose that there exists $\mathbf{v} \in K$ such that

$$
(\tilde{\boldsymbol{\tau}}(t), \varepsilon(\mathbf{v}))_{Q}<0 .
$$


12 A frictionless contact problem for viscoelastic materials

Using (4.19), since $\lambda \mathbf{v} \in K$ for $\lambda \geq 0$, it follows that

$$
\lambda(\tilde{\boldsymbol{\tau}}(t), \varepsilon(\mathbf{v}))_{Q}>\alpha \quad \forall \lambda \geq 0
$$

and, passing to the limit when $\lambda \rightarrow+\infty$, from (4.21) we obtain $\alpha \leq-\infty$ which is in contradiction with $\alpha \in \mathbb{R}$. We conclude that

$$
(\tilde{\boldsymbol{\tau}}(t), \boldsymbol{\varepsilon}(\mathbf{v}))_{Q} \geq 0 \quad \forall \mathbf{v} \in K .
$$

Let $\tilde{\boldsymbol{\sigma}}(t)=\mathscr{A} \boldsymbol{\varepsilon}(\mathbf{f}(t)) \in Q$. Using (3.6) we find

$$
(\tilde{\boldsymbol{\sigma}}(t), \boldsymbol{\varepsilon}(\mathbf{v}))_{Q}=(\mathbf{f}(t), \mathbf{v})_{V} \quad \forall \mathbf{v} \in V .
$$

It follows from (3.9), (4.23), and (4.24) that $\tilde{\boldsymbol{\tau}}(t)+\tilde{\boldsymbol{\sigma}}(t) \in \Sigma(t)$. Taking $\boldsymbol{\tau}=\tilde{\boldsymbol{\tau}}(t)+\tilde{\boldsymbol{\sigma}}(t)$ in $(3.15)$ we find

$$
(\tilde{\boldsymbol{\tau}}(t), \boldsymbol{\varepsilon}(\mathbf{u}(t)))_{Q} \geq(\boldsymbol{\sigma}(t)-\tilde{\boldsymbol{\sigma}}(t), \boldsymbol{\varepsilon}(\mathbf{u}(t)))_{Q} .
$$

Keeping now in mind (4.19) and (4.20), from (4.25) we deduce

$$
(\sigma(t)-\tilde{\boldsymbol{\sigma}}(t), \varepsilon(\mathbf{u}(t)))_{Q}<0 .
$$

On the other hand, (3.9) and (4.24) imply that $2 \sigma(t)-\tilde{\boldsymbol{\sigma}}(t) \in \Sigma(t)$ and, taking $\boldsymbol{\tau}=2 \boldsymbol{\sigma}(t)-\tilde{\boldsymbol{\sigma}}(t)$ in (3.15), we obtain

$$
(\boldsymbol{\sigma}(t)-\tilde{\boldsymbol{\sigma}}(t), \boldsymbol{\varepsilon}(\mathbf{u}(t)))_{Q} \geq 0 .
$$

We note that (4.26) and (4.27) are in contradiction. Therefore, $\mathbf{u}(t) \in K$. Taking now $\boldsymbol{\tau}=\tilde{\boldsymbol{\sigma}}(t)$ in (3.15) and using (4.24) we have

$$
(\mathbf{f}(t), \mathbf{u}(t))_{V} \geq(\sigma(t), \varepsilon(\mathbf{u}(t)))_{Q} .
$$

As $\boldsymbol{\sigma}(t) \in \Sigma(t)$ and $\mathbf{u}(t) \in K$, from (3.9) it follows that

$$
(\boldsymbol{\sigma}(t), \boldsymbol{\varepsilon}(\mathbf{u}(t)))_{Q} \geq(\mathbf{f}(t), \mathbf{u}(t))_{V} .
$$

So, from (4.28) and (4.29) we obtain

$$
(\boldsymbol{\sigma}(t), \varepsilon(\mathbf{u}(t)))_{Q}=(\mathbf{f}(t), \mathbf{u}(t))_{V} .
$$

The inequality in (3.13) results now from (3.9) and (4.30), which concludes the proof. 


\section{A spatially semi-discrete scheme}

In this section, we consider an approximation of Problem 3.1 by discretizing only the spatial domain. First we observe that, in terms of displacements, Problem 3.1 can be equivalently stated as finding $\mathbf{u}:[0, T] \rightarrow$ $V$ such that the initial value condition (3.14) holds and for a.e. $t \in(0, T)$, $\mathbf{u}(t) \in K$, and

$$
\begin{gathered}
(\mathcal{A} \varepsilon(\dot{\mathbf{u}}(t)), \varepsilon(\mathbf{v})-\varepsilon(\mathbf{u}(t)))_{Q}+(\mathcal{G} \varepsilon(\mathbf{u}(t)), \varepsilon(\mathbf{v})-\varepsilon(\mathbf{u}(t)))_{Q} \\
\geq(\mathbf{f}(t), \mathbf{v}-\mathbf{u}(t))_{V} \quad \forall \mathbf{v} \in K .
\end{gathered}
$$

Let $V^{h}$ be a finite-dimensional subspace of $V$, which can be constructed for example by the finite-element method. Here $h \rightarrow 0+$ is a discretization parameter. Denote $K^{h}=V^{h} \cap K$. Notice that $K^{h}$ is a nonempty, closed, convex subset of $V^{h}$. Let $\mathbf{u}_{0}^{h} \in K^{h}$ be an approximation of $\mathbf{u}_{0}$. Then a spatially semi-discrete scheme of Problem 3.1 is the following.

Problem 5.1. Find a displacement field $\mathbf{u}^{h}:[0, T] \rightarrow V^{h}$ such that

$$
\mathbf{u}^{h}(0)=\mathbf{u}_{0}^{h}
$$

and for a.e. $t \in(0, T), \mathbf{u}^{h}(t) \in K^{h}$, and

$$
\begin{aligned}
\left(\mathcal{A} \varepsilon\left(\dot{\mathbf{u}}^{h}(t)\right), \varepsilon\left(\mathbf{v}^{h}\right)-\right. & \left.\varepsilon\left(\mathbf{u}^{h}(t)\right)\right)_{Q}+\left(\mathcal{G} \varepsilon\left(\mathbf{u}^{h}(t)\right), \varepsilon\left(\mathbf{v}^{h}\right)-\varepsilon\left(\mathbf{u}^{h}(t)\right)\right)_{Q} \\
& \geq\left(\mathbf{f}(t), \mathbf{v}^{h}-\mathbf{u}^{h}(t)\right)_{V} \quad \forall \mathbf{v}^{h} \in K^{h} .
\end{aligned}
$$

We first show the existence of a unique solution to Problem 5.1 by an argument similar to the proof of Theorem 4.1.

Theorem 5.2. Assume (3.1), (3.2), (3.3), and (3.4). Then there exists a unique semi-discrete solution $\mathbf{u} \in W^{1, \infty}\left(0, T ; V^{h}\right)$ to Problem 5.1 .

Proof. We apply Theorem 2.2 for $X=V^{h}$ with the inner product $(\cdot, \cdot)_{V}$. Denote by $I^{h}: V^{h} \rightarrow V^{h}$ the identity operator on $V^{h}$. We define an operator $B^{h}: V^{h} \rightarrow V^{h}$ by

$$
\left(B^{h} \mathbf{u}^{h}, \mathbf{v}^{h}\right)_{V}=\left(\mathcal{G} \varepsilon\left(\mathbf{u}^{h}\right), \varepsilon\left(\mathbf{v}^{h}\right)\right)_{Q} \quad \forall \mathbf{u}^{h}, \mathbf{v}^{h} \in V^{h} .
$$

Then as in the proof of Theorem 4.1,

$$
\left\|B^{h} \mathbf{u}_{1}^{h}-B^{h} \mathbf{u}_{2}^{h}\right\|_{V} \leq \frac{\mathcal{L}_{\mathcal{G}}}{m_{\mathcal{A}}}\left\|\mathbf{u}_{1}^{h}-\mathbf{u}_{2}^{h}\right\|_{V} \quad \forall \mathbf{u}_{1}^{h}, \mathbf{u}_{2}^{h} \in V^{h} .
$$

So the operator

$$
B^{h}+\frac{\mathcal{L}_{\mathcal{G}}}{m_{\mathcal{A}}} I^{h}: V^{h} \longrightarrow V^{h}
$$


14 A frictionless contact problem for viscoelastic materials

is monotone and Lipschitz continuous. For the indicator function denoted $\psi_{K^{h}}^{h}: V^{h} \rightarrow(-\infty, \infty]$ defined by

$$
\psi_{K^{h}}^{h}\left(\mathbf{v}^{h}\right)= \begin{cases}0 & \text { if } \mathbf{v}^{h} \in K^{h}, \\ \infty & \text { otherwise, }\end{cases}
$$

we denote its (discrete) subdifferential

$$
\partial^{h} \psi_{K^{h}}^{h}\left(\mathbf{u}^{h}\right)=\left\{\mathbf{g}^{h} \in V^{h} \mid\left(\mathbf{g}^{h}, \mathbf{v}^{h}-\mathbf{u}^{h}\right)_{V} \leq 0 \forall \mathbf{v}^{h} \in K^{h}\right\},
$$

for any $\mathbf{u}^{h} \in K^{h}$. Like the subdifferential $\partial \psi_{K}, \partial^{h} \psi_{K^{h}}^{h}$ is a maximal monotone operator on $V^{h}$ and the effective domain of $\partial^{h} \psi_{K^{h}}^{h}$ is $K^{h}$. We then define $\mathbf{f}^{h}:[0, T] \rightarrow V^{h}$ by

$$
\left(\mathbf{f}^{h}(t), \mathbf{v}^{h}\right)_{V}=\left(\mathbf{f}(t), \mathbf{v}^{h}\right)_{V} \quad \forall \mathbf{v}^{h} \in V^{h} .
$$

We have the regularity $\mathbf{f}^{h} \in W^{1,1}\left(0, T ; V^{h}\right)$. Applying Theorem 2.2 with $X=V^{h}, A^{h}=\partial^{h} \psi_{K^{h}}^{h}+B^{h}$, and $\mathbf{f}^{h}$ defined in (5.9), we obtain the existence of a unique $\mathbf{u}^{h} \in W^{1, \infty}\left(0, T ; V^{h}\right)$ such that (5.2) and

$$
\dot{\mathbf{u}}^{h}(t)+\partial^{h} \psi_{K^{h}}^{h}\left(\mathbf{u}^{h}(t)\right)+B^{h} \mathbf{u}^{h}(t) \ni \mathbf{f}^{h}(t) \quad \text { a.e. } t \in(0, T)
$$

hold. It is easy to see that (5.10) is equivalent to $\mathbf{u}^{h}(t) \in K^{h}$ and inequality (5.3) for a.e. $t \in(0, T)$.

Now we turn to an error analysis of the method. We take $\mathbf{v}=\mathbf{u}^{h}(s)$ in (5.1) and add it to (5.3) with $\mathbf{v}^{h}=\mathbf{v}^{h}(s) \in K^{h}$. After some rearrangement of the terms, we obtain

$$
\begin{aligned}
&\left(\mathcal{A} \varepsilon(\dot{\mathbf{u}}(s))-\mathcal{A} \varepsilon\left(\dot{\mathbf{u}}^{h}(s)\right), \varepsilon\left(\mathbf{u}(s)-\mathbf{u}^{h}(s)\right)\right)_{Q} \\
& \leq\left(\mathcal{A} \varepsilon\left(\dot{\mathbf{u}}^{h}(s)\right), \varepsilon\left(\mathbf{v}^{h}-\mathbf{u}(s)\right)\right)_{Q} \\
&+\left(\mathcal{G} \varepsilon(\mathbf{u}(s))-\mathcal{G} \varepsilon\left(\mathbf{u}^{h}(s)\right), \varepsilon\left(\mathbf{u}^{h}(s)-\mathbf{u}(s)\right)\right)_{Q} \\
&+\left(\mathcal{G} \varepsilon\left(\mathbf{u}^{h}(s)\right), \varepsilon\left(\mathbf{v}^{h}-\mathbf{u}(s)\right)\right)_{Q}-\left(\mathbf{f}(s), \mathbf{v}^{h}-\mathbf{u}^{h}(s)\right)_{V} \quad \text { a.e. } s \in(0, T) .
\end{aligned}
$$

Using now (3.6), we have

$$
\begin{aligned}
\frac{1}{2} \frac{d}{d s}\left\|\mathbf{u}(s)-\mathbf{u}^{h}(s)\right\|_{V}^{2} \leq & \left(\mathcal{A} \varepsilon\left(\dot{\mathbf{u}}^{h}(s)-\dot{\mathbf{u}}(s)\right), \boldsymbol{\varepsilon}\left(\mathbf{v}^{h}-\mathbf{u}(s)\right)\right)_{Q} \\
& +\left(\mathcal{G} \varepsilon\left(\mathbf{u}^{h}(s)\right)-\mathcal{G} \varepsilon(\mathbf{u}(s)), \varepsilon\left(\mathbf{v}^{h}-\mathbf{u}(s)\right)\right)_{Q} \\
& +\left(\mathcal{G} \varepsilon(\mathbf{u}(s))-\mathcal{G}_{\boldsymbol{G}} \varepsilon\left(\mathbf{u}^{h}(s)\right), \boldsymbol{\varepsilon}\left(\mathbf{u}^{h}(s)-\mathbf{u}(s)\right)\right)_{Q} \\
& +R\left(s ; \mathbf{v}^{h}-\mathbf{u}(s)\right) \quad \text { a.e. } s \in(0, T),
\end{aligned}
$$


where

$$
R(s ; \mathbf{v})=(\mathcal{A} \varepsilon(\dot{\mathbf{u}}(s)), \boldsymbol{\varepsilon}(\mathbf{v}))_{Q}+(\mathcal{G} \varepsilon(\mathbf{u}(s)), \varepsilon(\mathbf{v}))_{Q}-(\mathbf{f}(s), \mathbf{v})_{V}
$$

for all $\mathbf{v} \in V$, a.e. $s \in(0, T)$.

Let $t \in[0, T]$, integrating inequality (5.12) from 0 to $t$ and using the initial conditions (3.14) and (5.2), we find

$$
\begin{aligned}
\frac{1}{2}\left[\left\|\mathbf{u}(t)-\mathbf{u}^{h}(t)\right\|_{V}^{2}-\left\|\mathbf{u}_{0}-\mathbf{u}_{0}^{h}\right\|_{V}^{2}\right] \\
\leq \int_{0}^{t}\left(\mathcal{A} \boldsymbol{\varepsilon}\left(\dot{\mathbf{u}}^{h}(s)-\dot{\mathbf{u}}(s)\right), \boldsymbol{\varepsilon}\left(\mathbf{v}^{h}-\mathbf{u}(s)\right)\right)_{Q} d s \\
\quad+\int_{0}^{t} \mathcal{L}_{\mathcal{G}}\left\|\mathbf{u}(s)-\mathbf{u}^{h}(s)\right\|_{V}\left\|\mathbf{u}(s)-\mathbf{v}^{h}\right\|_{V} d s \\
\quad+\int_{0}^{t} \mathcal{L}_{\mathcal{G}}\left\|\mathbf{u}(s)-\mathbf{u}^{h}(s)\right\|_{V}^{2} d t+\int_{0}^{t}\left|R\left(s ; \mathbf{v}^{h}-\mathbf{u}(s)\right)\right| d s .
\end{aligned}
$$

We perform an integration by parts on the first term in the right-hand side

$$
\begin{aligned}
\int_{0}^{t} & \left(\mathcal{A} \varepsilon\left(\dot{\mathbf{u}}^{h}(s)-\dot{\mathbf{u}}(s)\right), \boldsymbol{\varepsilon}\left(\mathbf{v}^{h}-\mathbf{u}(s)\right)\right)_{Q} d s \\
= & \left(\mathcal{A} \varepsilon\left(\mathbf{u}(t)-\mathbf{u}^{h}(t)\right), \varepsilon\left(\mathbf{u}(t)-\mathbf{v}^{h}(t)\right)\right)_{Q}-\left(\mathcal{A} \varepsilon\left(\mathbf{u}_{0}-\mathbf{u}_{0}^{h}\right), \varepsilon\left(\mathbf{u}_{0}-\mathbf{v}_{0}^{h}\right)\right)_{Q} \\
& \quad-\int_{0}^{t}\left(\mathcal{A} \varepsilon\left(\mathbf{u}^{h}(s)-\mathbf{u}(s)\right), \varepsilon\left(\dot{\mathbf{v}}^{h}-\dot{\mathbf{u}}(s)\right)\right)_{Q} d s .
\end{aligned}
$$

Then we have

$$
\begin{aligned}
\left\|\mathbf{u}(t)-\mathbf{u}^{h}(t)\right\|_{V}^{2} \leq & c\left(\left\|\mathbf{u}(t)-\mathbf{v}^{h}(t)\right\|_{V}^{2}+\left\|\mathbf{u}_{0}-\mathbf{u}_{0}^{h}\right\|_{V}^{2}+\left\|\mathbf{u}_{0}-\mathbf{v}_{0}^{h}\right\|_{V}^{2}\right) \\
& +c \int_{0}^{t}\left\|\mathbf{u}(s)-\mathbf{u}^{h}(s)\right\|_{V}^{2} d s+c \int_{0}^{t}\left\|\mathbf{u}(s)-\mathbf{v}^{h}(s)\right\|_{V}^{2} d s \\
& +c \int_{0}^{t}\left\|\dot{\mathbf{u}}(s)-\dot{\mathbf{v}}^{h}(s)\right\|_{V}^{2} d s+c \int_{0}^{t}\left|R\left(s ; \mathbf{v}^{h}(s)-\mathbf{u}(s)\right)\right| d s .
\end{aligned}
$$

Here and below, $c$ denotes various positive generic constants which do not depend on $h$ and whose values may change from line to line. Since

$$
\mathbf{u}(t)-\mathbf{v}^{h}(t)=\mathbf{u}_{0}-\mathbf{v}_{0}^{h}+\int_{0}^{t}\left(\dot{\mathbf{u}}(s)-\dot{\mathbf{v}}^{h}(s)\right) d s,
$$


16 A frictionless contact problem for viscoelastic materials

we have

$$
\begin{array}{r}
\left\|\mathbf{u}(t)-\mathbf{v}^{h}(t)\right\|_{V}^{2} \leq c\left\|\mathbf{u}_{0}-\mathbf{v}_{0}^{h}\right\|_{V}^{2}+c \int_{0}^{t}\left\|\dot{\mathbf{u}}(s)-\dot{\mathbf{v}}^{h}(s)\right\|_{V}^{2} d s, \\
\int_{0}^{t}\left\|\mathbf{u}(s)-\mathbf{v}^{h}(s)\right\|_{V}^{2} d s \leq c\left\|\mathbf{u}_{0}-\mathbf{v}_{0}^{h}\right\|_{V}^{2}+c \int_{0}^{t}\left\|\dot{\mathbf{u}}(s)-\dot{\mathbf{v}}^{h}(s)\right\|_{V}^{2} d s .
\end{array}
$$

Thus,

$$
\begin{aligned}
\left\|\mathbf{u}(t)-\mathbf{u}^{h}(t)\right\|_{V}^{2} \leq & c\left(\left\|\mathbf{u}_{0}-\mathbf{u}_{0}^{h}\right\|_{V}^{2}+\left\|\mathbf{u}_{0}-\mathbf{v}_{0}^{h}\right\|_{V}^{2}\right)+c \int_{0}^{t}\left\|\mathbf{u}(s)-\mathbf{u}^{h}(s)\right\|_{V}^{2} d s \\
& +c \int_{0}^{t}\left\|\dot{\mathbf{u}}(s)-\dot{\mathbf{v}}^{h}(s)\right\|_{V}^{2} d s+c \int_{0}^{t}\left|R\left(s ; \mathbf{v}^{h}(s)-\mathbf{u}(s)\right)\right| d s .
\end{aligned}
$$

Applying Gronwall's inequality, we obtain

$$
\begin{aligned}
\left\|\mathbf{u}-\mathbf{u}^{h}\right\|_{L^{\infty}(0, T ; V)}^{2} \leq c( & \left\|\mathbf{u}_{0}-\mathbf{v}_{0}^{h}\right\|_{V}^{2}+\left\|\dot{\mathbf{u}}-\dot{\mathbf{v}}^{h}\right\|_{L^{2}(0, T ; V)}^{2} \\
& \left.+\left\|R\left(\cdot ; \mathbf{v}^{h}-\mathbf{u}\right)\right\|_{L^{1}(0, T ; V)}+\left\|\mathbf{u}_{0}-\mathbf{u}_{0}^{h}\right\|_{V}^{2}\right) .
\end{aligned}
$$

Since $\mathbf{v}^{h} \in W^{1,2}\left(0, T ; K^{h}\right)$ is arbitrary, we then have

$$
\begin{aligned}
& \left\|\mathbf{u}-\mathbf{u}^{h}\right\|_{L^{\infty}(0, T ; V)} \\
& \leq \inf _{\mathbf{v}^{h} \in W^{1,2}\left(0, T ; K^{h}\right)}\left(\left\|\mathbf{u}_{0}-\mathbf{v}_{0}^{h}\right\|_{V}+\left\|\dot{\mathbf{u}}-\dot{\mathbf{v}}^{h}\right\|_{L^{2}(0, T ; V)}\right. \\
& \left.\quad+\left\|R\left(\cdot ; \mathbf{v}^{h}-\mathbf{u}\right)\right\|_{L^{1}(0, T ; V)}^{1 / 2}\right)+c\left\|\mathbf{u}_{0}-\mathbf{u}_{0}^{h}\right\|_{V} .
\end{aligned}
$$

Inequality (5.21) is a basis for convergence analysis and error estimation. For definiteness, in the following we consider the two-dimensional case. We assume $\Omega$ is a polygon. Then the boundary $\partial \Omega$ consists of line segments. Write

$$
\bar{\Gamma}_{3}=\cup_{i=1}^{I} \bar{\Gamma}_{3, i}
$$

with each $\bar{\Gamma}_{3, i}$ being a line segment. Let $\left\{\tau_{h}\right\}_{h}$ be a family of regular finite-element partitions of $\bar{\Omega}$ into triangles (cf. [2]), compatible to the boundary decomposition $\partial \Omega=\bar{\Gamma}_{1} \cap \bar{\Gamma}_{2} \cap \bar{\Gamma}_{3}$, that is, any point when the boundary condition type changes is a vertex of the partitions. Let $\left\{V^{h}\right\}_{h}$ $c V$ be the corresponding family of finite-element spaces of linear elements which are zero on $\bar{\Gamma}_{1}$. Then $K^{h}=K \cap V^{h}$ consists of functions $\mathbf{v}^{h} \in C(\bar{\Omega})^{2}$ such that on each element, $\mathbf{v}^{h}$ is an affine function, $\mathbf{v}^{h}(\mathbf{z})=\mathbf{0}$ for any vertex $\mathbf{z} \in \bar{\Gamma}_{1}$, and $v_{v}^{h}(\mathbf{z}) \leq 0$ for any vertex $\mathbf{z} \in \bar{\Gamma}_{3}$. Let $\Pi^{h}$ : $C(\bar{\Omega})^{2} \rightarrow V^{h}$ be the finite-element interpolation operator. Then we have the interpolation error estimate (cf. [2]):

$$
\left\|\mathbf{v}-\Pi^{h} \mathbf{v}\right\|_{V} \leq c h|\mathbf{v}|_{H^{2}(\Omega)} \quad \forall \mathbf{v} \in H^{2}(\Omega)^{2} .
$$


The restriction of the partitions $\left\{\tau_{h}\right\}_{h}$ on $\bar{\Gamma}_{3}$ induces a regular family of finite-element partitions of $\bar{\Gamma}_{3}$. So we also have the interpolation error estimate

$$
\left\|v-\Pi^{h} v\right\|_{L^{2}\left(\Gamma_{3, i}\right)} \leq c h|v|_{H^{2}\left(\Gamma_{3, i}\right)} \quad \forall v \in H^{2}\left(\Gamma_{3, i}\right)^{2}, 1 \leq i \leq I .
$$

We notice that for $\mathbf{v} \in K \cap C(\bar{\Omega}), \Pi^{h} \mathbf{v} \in K^{h}$.

We have the following convergence result under the basic solution regularity.

Proposition 5.3. Assume the intersection $\bar{\Gamma}_{1} \cap \bar{\Gamma}_{3}$ has a finite number of points. If we choose $\mathbf{u}_{0}^{h} \in K^{h}$ such that

$$
\left\|\mathbf{u}_{0}-\mathbf{u}_{0}^{h}\right\|_{V} \longrightarrow 0 \quad \text { as } h \longrightarrow 0 .
$$

Then the numerical method converges, that is,

$$
\left\|\mathbf{u}-\mathbf{u}^{h}\right\|_{L^{\infty}(0, T ; V)} \longrightarrow 0 \quad \text { as } h \longrightarrow 0 .
$$

Proof. From definition (5.13), we immediately get

$$
|R(s ; \mathbf{v})| \leq c\left(\|\mathbf{u}\|_{W^{1, \infty}(0, T ; V)}+\|\mathbf{f}\|_{L^{\infty}(0, T ; V)}\right)\|\mathbf{v}\|_{V} \quad \forall \mathbf{v} \in V \text {, a.e. } s \in(0, T) .
$$

Thus, we derive from (5.21) that

$$
\begin{aligned}
\left\|\mathbf{u}-\mathbf{u}^{h}\right\|_{L^{\infty}(0, T ; V)} & \\
\leq \inf _{\mathbf{v}^{h} \in W^{1,2}\left(0, T ; K^{h}\right)} & \left(\left\|\mathbf{u}_{0}-\mathbf{v}_{0}^{h}\right\|_{V}+\left\|\dot{\mathbf{u}}-\dot{\mathbf{v}}^{h}\right\|_{L^{2}(0, T ; V)}\right. \\
& \left.+\left\|\mathbf{u}-\mathbf{v}^{h}\right\|_{L^{\infty}(0, T ; V)}^{1 / 2}\right)+c\left\|\mathbf{u}_{0}-\mathbf{u}_{0}^{h}\right\|_{V} .
\end{aligned}
$$

With the assumption made on the boundary, it is known (see [14]) that $K \cap C^{\infty}(\bar{\Omega})^{2}$ is dense in $K$ with respect to the norm of $V$. It can then be shown (see [9]) that $W^{1,2}\left(0, T ; K \cap C^{\infty}(\bar{\Omega})^{2}\right)$ is dense in $W^{1,2}(0, T ; K)$ with respect to the norm of $W^{1,2}(0, T ; V)$. So for any $\varepsilon>0$, there exists $\mathbf{u}_{\varepsilon} \in W^{1,2}\left(0, T ; K \cap C^{\infty}(\bar{\Omega})^{2}\right)$ such that

$$
\left\|\mathbf{u}-\mathbf{u}_{\varepsilon}\right\|_{W^{1,2}(0, T ; V)}<\varepsilon .
$$

For any $t \in[0, T]$, let $\mathbf{v}^{h}(t)=\Pi^{h} \mathbf{u}_{\varepsilon}(t)$. Then $\mathbf{v}^{h}(t) \in K^{h}, \dot{\mathbf{v}}^{h}(t)=\Pi^{h} \dot{\mathbf{u}}_{\varepsilon}(t)$, and by (5.23) we obtain

$$
\begin{aligned}
& \left\|\mathbf{u}_{\varepsilon}(t)-\Pi^{h} \mathbf{u}_{\varepsilon}(t)\right\|_{V} \leq c h\left\|\mathbf{u}_{\varepsilon}(t)\right\|_{H^{2}(\Omega)^{2}}, \\
& \left\|\dot{\mathbf{u}}_{\varepsilon}(t)-\Pi^{h} \dot{\mathbf{u}}_{\varepsilon}(t)\right\|_{V} \leq c h\left\|\dot{\mathbf{u}}_{\varepsilon}(t)\right\|_{H^{2}(\Omega)^{2}} .
\end{aligned}
$$


18 A frictionless contact problem for viscoelastic materials

Then

$$
\begin{aligned}
& \left\|\mathbf{u}_{\varepsilon}-\Pi^{h} \mathbf{u}_{\varepsilon}\right\|_{L^{2}(0, T ; V)} \leq c h\left\|\mathbf{u}_{\varepsilon}\right\|_{L^{\infty}\left(0, T ; H^{2}(\Omega)^{2}\right)^{\prime}} \\
& \left\|\dot{\mathbf{u}}_{\varepsilon}-\Pi^{h} \dot{\mathbf{u}}_{\varepsilon}\right\|_{L^{2}(0, T ; V)} \leq c h\left\|\dot{\mathbf{u}}_{\varepsilon}\right\|_{L^{\infty}\left(0, T ; H^{2}(\Omega)^{2}\right)} .
\end{aligned}
$$

Also we have

$$
\left\|\mathbf{u}_{\varepsilon}(0)-\Pi^{h} \mathbf{u}_{\varepsilon}(0)\right\|_{V} \leq c h\left\|\mathbf{u}_{\varepsilon}(0)\right\|_{H^{2}(\Omega)^{2}} .
$$

Applying the triangle inequality for the norm, we then obtain from (5.28) that

$$
\begin{aligned}
\| \mathbf{u}-\mathbf{u}^{h} & \|_{L^{\infty}(0, T ; V)} \\
\leq & c\left(\varepsilon+\varepsilon^{1 / 2}\right)+c h\left(\left\|\mathbf{u}_{\varepsilon}(0)\right\|_{H^{2}(\Omega)^{2}}+\left\|\dot{\mathbf{u}}_{\varepsilon}\right\|_{L^{2}\left(0, T ; H^{2}(\Omega)^{2}\right)}\right) \\
& +c h^{1 / 2}\left\|\mathbf{u}_{\varepsilon}\right\|_{L^{\infty}\left(0, T ; H^{2}(\Omega)^{2}\right)}^{1 / 2}+c\left\|\mathbf{u}_{0}-\mathbf{u}_{0}^{h}\right\|_{V} .
\end{aligned}
$$

We can choose $h$ sufficiently small so that

$$
\begin{aligned}
h\left(\| \mathbf{u}_{\varepsilon}(0)\right. & \left.\left\|_{H^{2}(\Omega)^{2}}+\right\| \dot{\mathbf{u}}_{\varepsilon} \|_{L^{2}\left(0, T ; H^{2}(\Omega)^{2}\right)}\right) \\
& +h^{1 / 2}\left\|\mathbf{u}_{\varepsilon}\right\|_{L^{\infty}\left(0, T ; H^{2}(\Omega)^{2}\right)}^{1 / 2}+\left\|\mathbf{u}_{0}-\mathbf{u}_{0}^{h}\right\|_{V}<\varepsilon .
\end{aligned}
$$

Then

$$
\left\|\mathbf{u}-\mathbf{u}^{h}\right\|_{L^{\infty}(0, T ; V)} \leq c\left(\varepsilon+\varepsilon^{1 / 2}\right) .
$$

Therefore, we have the convergence (5.26).

We remark that assumption (5.25) is satisfied if $\mathbf{u}_{0}^{h}=\Pi^{h} \mathbf{u}_{0}$ when $\mathbf{u}_{0} \in$ $C(\bar{\Omega})$.

Now, we provide an optimal error estimates result under additional regularity on the solution.

Proposition 5.4. Assume (4.12) and

$$
\mathbf{u} \in W^{1,2}\left(0, T ; H^{2}(\Omega)^{d}\right),\left.\quad u_{v}\right|_{\Gamma_{3, i}} \in L^{1}\left(0, T ; H^{2}\left(\Gamma_{3, i}\right)\right), \quad 1 \leq i \leq I .
$$

We choose $\mathbf{u}_{0}^{h}=\Pi^{h} \mathbf{u}_{0}$. Then we have the following optimal order error estimate:

$$
\left\|\mathbf{u}-\mathbf{u}^{h}\right\|_{L^{\infty}(0, T ; V)}=O(h) .
$$

Proof. We first derive a sharper bound on the residual term under assumption (4.12). Let $\mathbf{v} \in V$. The equalities and inequalities below involving the argument $t$ are understood to be valid for almost any $t \in(0, T)$. Using (5.13) and (3.12) we obtain

$$
R(t ; \mathbf{v})=(\boldsymbol{\sigma}(t), \boldsymbol{\varepsilon}(\mathbf{v}))_{Q}-(\mathbf{f}(t), \mathbf{v})_{V} \quad \forall \mathbf{v} \in V \text {, a.e. } t \in(0, T) .
$$


Performing an integration by parts on the first term and using (3.7), we have

$$
R(t ; \mathbf{v})=\int_{\Gamma} \boldsymbol{\sigma}(t) \boldsymbol{v} \cdot \mathbf{v} d a-\int_{\Omega} \operatorname{Div} \boldsymbol{\sigma}(t) \cdot \mathbf{v} d x-\int_{\Omega} \mathbf{f}_{0}(t) \cdot \mathbf{v} d x-\int_{\Gamma_{2}} \mathbf{f}_{2}(t) \cdot \mathbf{v} d a
$$

Using now the relations (4.11) and (4.13), we obtain

$$
R(t ; \mathbf{v})=\int_{\Gamma_{3}} \sigma_{v}(t) v_{v} d a
$$

Thus, instead of (5.27), we have the following bound:

$$
|R(t ; \mathbf{v})| \leq c\left\|\sigma_{v}\right\|_{L^{\infty}\left(0, T ; L^{2}(\Gamma)\right)}\left\|v_{v}\right\|_{L^{2}\left(\Gamma_{3}\right)} .
$$

By (5.21) we then get

$$
\begin{aligned}
\left\|\mathbf{u}-\mathbf{u}^{h}\right\|_{L^{\infty}(0, T ; V)} & \\
\leq \operatorname{linf}_{\mathbf{v}^{h} \in W^{1,2}\left(0, T ; K^{h}\right)} & \left(\left\|\mathbf{u}_{0}-\mathbf{v}_{0}^{h}\right\|_{V}+\left\|\dot{\mathbf{u}}-\dot{\mathbf{v}}^{h}\right\|_{L^{2}(0, T ; V)}\right. \\
& \left.+\left\|u_{v}-v_{v}^{h}\right\|_{L^{1}\left(0, T ; L^{2}\left(\Gamma_{3}\right)\right)}^{1 / 2}\right)+c\left\|\mathbf{u}_{0}-\Pi^{h} \mathbf{u}_{0}\right\|_{V} .
\end{aligned}
$$

For any $t \in[0, T]$, let $\mathbf{v}^{h}(t)=\Pi^{h} \mathbf{u}(t)$ be the finite-element interpolant of $\mathbf{u}(t)$. Then we have $\Pi^{h} \mathbf{u} \in W^{1,2}\left(0, T ; K^{h}\right)$ and the interpolation error estimates

$$
\begin{gathered}
\left\|\mathbf{u}_{0}-\Pi^{h} \mathbf{u}_{0}\right\|_{V} \leq c h\left\|\mathbf{u}_{0}\right\|_{H^{2}(\Omega)^{2}} \\
\left\|\dot{\mathbf{u}}-\Pi^{h} \dot{\mathbf{u}}\right\|_{L^{2}(0, T ; V)} \leq c h\|\dot{\mathbf{u}}\|_{L^{2}\left(0, T ; H^{2}(\Omega)^{2}\right)^{\prime}} \\
\left\|u_{v}-\Pi^{h} u_{v}\right\|_{L^{1}\left(0, T ; L^{2}\left(\Gamma_{3}\right)\right)} \leq c h^{2} \sum_{i=1}^{I}\left\|u_{v}\right\|_{L^{1}\left(0, T ; L^{2}\left(\Gamma_{3, i}\right)\right)}
\end{gathered}
$$

Now the error estimate (5.37) follows from (5.42), keeping in mind the previous inequalities.

From the viewpoint of applications, it is more important to consider fully discrete schemes where discretization is introduced with respect to both time and space variables. For fully discrete schemes, existence of a unique solution is not difficult to prove. However, derivation of error estimates for fully discrete solutions remains an open problem.

\section{Acknowledgment}

The work of W. Han was supported by NSF/DARPA under Grant DMS9874015. 


\section{A frictionless contact problem for viscoelastic materials}

\section{References}

[1] V. Barbu, Optimal Control of Variational Inequalities, Research Notes in Mathematics, vol. 100, Pitman, Massachusetts, 1984.

[2] P. G. Ciarlet, The Finite Element Method for Elliptic Problems, Studies in Mathematics and Its Applications, vol. 4, North-Holland Publishing, Amsterdam, 1978.

[3] S. Drabla, M. Rochdi, and M. Sofonea, A frictionless contact problem for elasticviscoplastic materials with internal state variables, Math. Comput. Modelling 26 (1997), no. 12, 31-47.

[4] G. Duvaut and J.-L. Lions, Inequalities in Mechanics and Physics, Grundlehren der mathematischen Wissenschaften, vol. 219, Springer-Verlag, Berlin, 1976.

[5] J. R. Fernandez-Garcia, W. Han, M. Shillor, and M. Sofonea, Recent advances in variational and numerical analysis of quasistatic contact problems, Proceedings of Fourteenth International Symposium on Mathematical Theory of Networks and Systems (MTNS 2000), 19-23 June 2000, Perpignan, France.

[6] G. Fichera, Problemi elastostatici con vincoli unilaterali: Il problema di Signorini con ambigue condizioni al contorno, Atti Accad. Naz. Lincei Mem. Cl. Sci. Fis. Mat. Natur. Sez. Ia (8) 7 (1963/1964), 91-140 (Italian).

[7] _ Boundary value problems of elasticity with unilateral constraints, Encyclopedia of Physics (S. Flüge, ed.), vol. 6a/2, Springer, Berlin, 1972.

[8] W. Han and M. Sofonea, Numerical analysis of a frictionless contact problem for elastic-viscoplastic materials, Comput. Methods Appl. Mech. Engrg. 190 (2000), no. 1-2, 179-191.

[9] - Quasistatic Contact Problems in Viscoelasticity and Viscoplasticity, American Mathematical Society and International Press, 2002, in press.

[10] J. Haslinger and I. Hlaváček, Contact between elastic bodies. I. Continuous problems, Apl. Mat. 25 (1980), no. 5, 324-347.

[11]_ Contact between elastic bodies. II. Finite element analysis, Apl. Mat. 26 (1981), no. 4, 263-290.

[12] Contact between elastic bodies. III. Dual finite element analysis, Apl. Mat. 26 (1981), no. 5, 321-344.

[13] I. Hlaváček, J. Haslinger, J. Nečas, and J. Lovíšek, Solution of Variational Inequalities in Mechanics, Applied Mathematical Sciences, vol. 66, SpringerVerlag, New York, 1988.

[14] I. Hlaváček and J. Lovíšek, A finite element analysis for the Signorini problem in plane elastostatics, Apl. Mat. 22 (1977), no. 3, 215-228.

[15] I. R. Ionescu and M. Sofonea, Functional and Numerical Methods in Viscoplasticity, Oxford University Press, New York, 1993.

[16] J. Jarušek, Dynamical contact problems for bodies with a singular memory, Boll. Un. Mat. Ital. A (7) 9 (1995), no. 3, 581-592.

[17] , Remark to dynamic contact problems for bodies with a singular memory, Comment. Math. Univ. Carolin. 39 (1998), no. 3, 545-550.

[18] N. Kikuchi and J. T. Oden, Contact Problems in Elasticity: A Study of Variational Inequalities and Finite Element Methods, SIAM Studies in Applied Mathematics, vol. 8, SIAM, Philadelphia, 1988. 
[19] J. Nečas and I. Hlavaček, Mathematical Theory of Elastic and Elastoplastic Bodies: An Introduction, Studies in Applied Mechanics, vol. 3, Elsevier Scientific Publishing, Amsterdam, 1981.

[20] P. D. Panagiotopoulos, Inequality Problems in Mechanics and Applications, Birkhäuser, Massachusetts, 1985.

[21] M. Rochdi, M. Shillor, and M. Sofonea, A quasistatic contact problem with directional friction and damped response, Appl. Anal. 68 (1998), no. 3-4, 409-422.

[22] Quasistatic viscoelastic contact with normal compliance and friction, J. Elasticity 51 (1998), no. 2, 105-126.

[23] M. Rochdi and M. Sofonea, On frictionless contact between two elasticviscoplastic bodies, Quart. J. Mech. Appl. Math. 50 (1997), no. 3, 481-496.

[24] A. Signorini, Sopra alcune questioni di elastostatica, Atti Soc. Ial. Progr. Sci., 1933.

[25] M. Sofonea, On a contact problem for elastic-viscoplastic bodies, Nonlinear Anal. 29 (1997), no. 9, 1037-1050.

Mikäel Barboteu: Laboratoire de Théorie des Systèmes, Université de Perpignan, 52 Avenue de Villeneuve, 66860 Perpignan, France

E-mail address: barboteu@univ-perp.fr

Weimin Han: Department of Mathematics, University of Iowa, Iowa City, IA 52242, USA

E-mail address: whan@math.uiowa.edu

Mircea Sofonea: Laboratoire de Théorie des Systèmes, Université de Perpignan, 52 Avenue de Villeneuve, 66860 Perpignan, France

E-mail address: sofonea@univ-perp.fr 


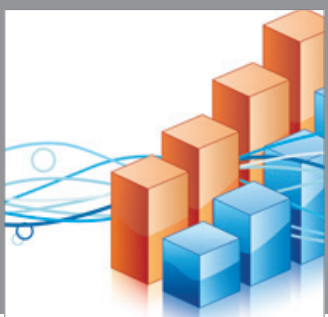

Advances in

Operations Research

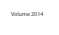

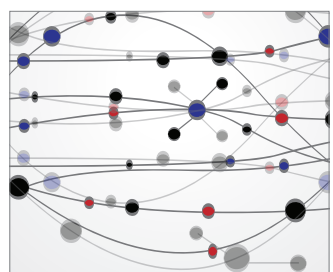

\section{The Scientific} World Journal
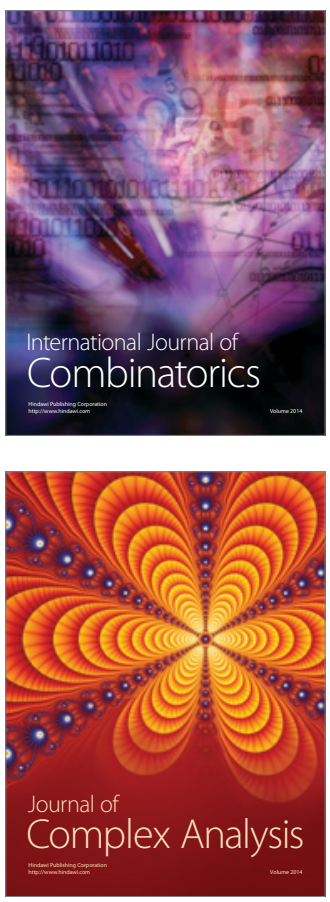

International Journal of

Mathematics and

Mathematical

Sciences
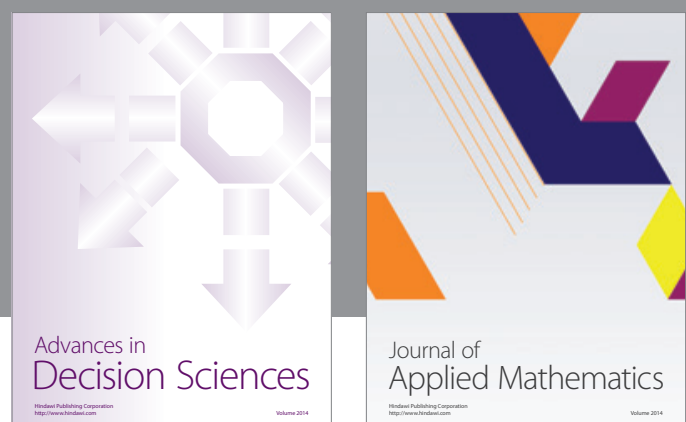

Journal of

Applied Mathematics
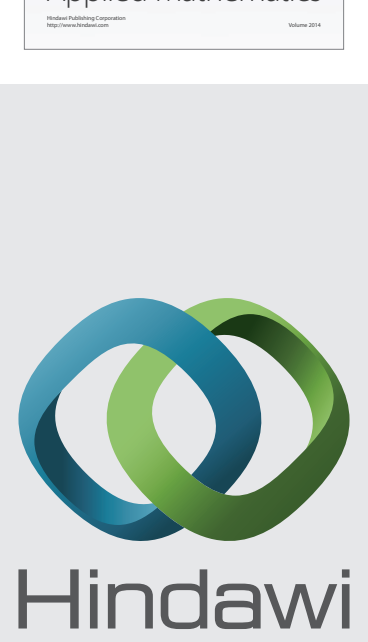

Submit your manuscripts at http://www.hindawi.com
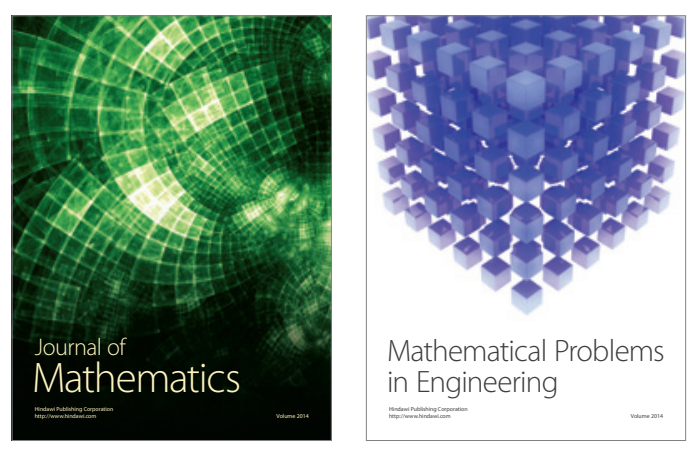

Mathematical Problems in Engineering
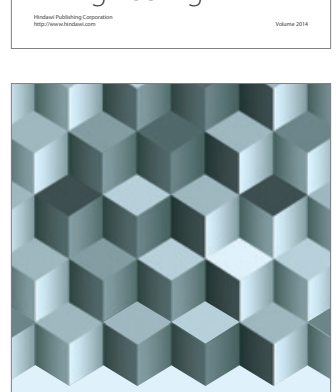

Journal of

Function Spaces
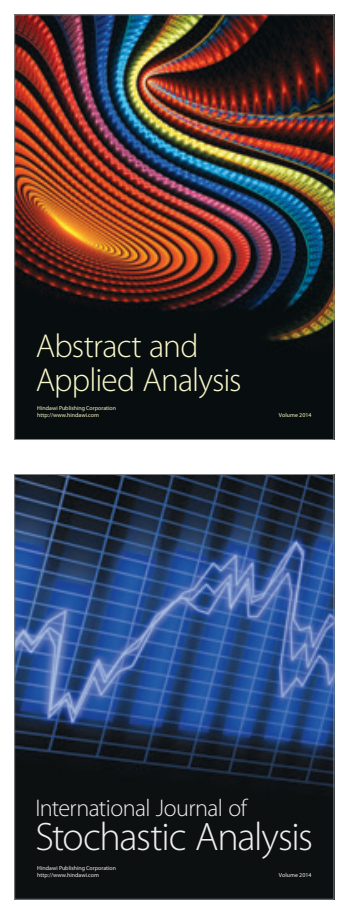

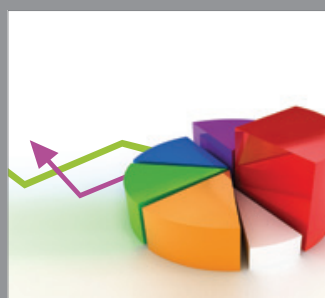

ournal of

Probability and Statistics

Promensencen
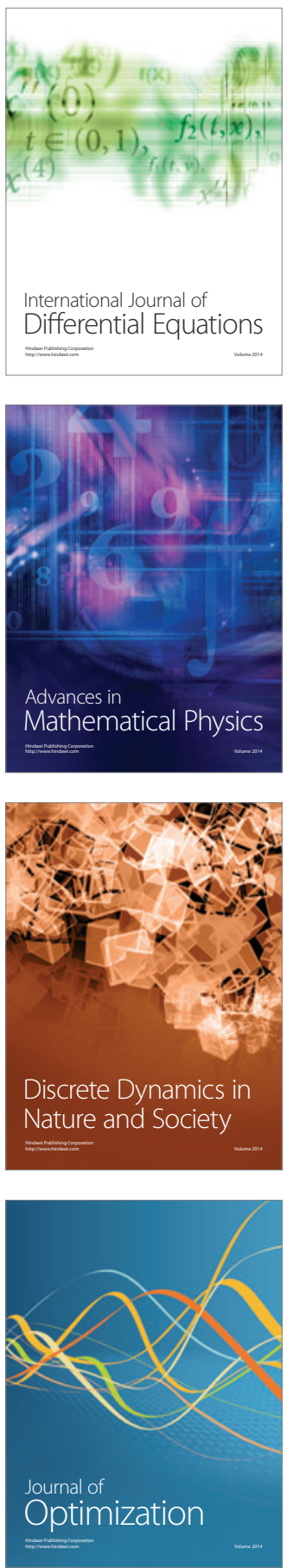